\title{
Faraday-Wave Contact-Line Shear Gradient Induces Streaming and Tracer Self-Organization: From Vortical to Hedgehoglike Patterns
}

\author{
Héctor Alarcón $\odot,{ }^{1,2, *}$ Matías Herrera-Muñoz $\odot,{ }^{3}$ Nicolas Périnet $\odot,{ }^{2}$ Nicolás Mujica $\odot,{ }^{2}$ \\ Pablo Gutiérrez $\mathbb{1}^{1, \dagger}$ and Leonardo Gordillo $\mathbb{1}^{3, *}$ \\ ${ }^{1}$ Instituto de Ciencias de la Ingeniería, Universidad de O'Higgins, Avenida Libertador Bernardo O'Higgins 611, Rancagua, Chile \\ ${ }^{2}$ Departamento de Física, Facultad de Ciencias Físicas y Matemáticas, Universidad de Chile, \\ Avenida Blanco Encalada 2008, Santiago, Chile \\ ${ }^{3}$ Departamento de Física, Universidad de Santiago de Chile, Avenida Ecuador 3493, Estación Central, Santiago, Chile
}

(Received 17 July 2020; accepted 4 November 2020; published 18 December 2020)

\begin{abstract}
In this Letter, we experimentally demonstrate self-organization of small tracers under the action of longitudinal Faraday waves in a narrow container. We observe a steady current formation dividing the interface in small cells given by Faraday-wave symmetries. These streaming currents rotate in each cell, and their circulation increases with wave amplitude. This streaming flow drives the tracers to form patterns, whose shapes depend on the Faraday-wave amplitude: From low to high amplitudes, we find tracers dispersed on vortices, narrow rotating rings, and a hedgehoglike pattern. We first describe the main pattern features and characterize the wave and tracers' motion. We then show experimentally that the main source of the streaming flow is the spatiotemporal-dependent shear at the wall contact line created by the Faraday wave itself. We end by presenting a $2 \mathrm{D}$ compressible advection model that considers the minimal ingredients present in the Faraday experiment, namely, the stationary circulation, the stretching component due to the oscillatory wave, and a steady converging field, which combined produce the observed self-organized patterns.
\end{abstract}

DOI: 10.1103/PhysRevLett.125.254505

Liquid interfaces are usually covered by particles. In the ocean, these include impurities, living organisms, nutrients, seeds, garbage, or even bubbles. These tracers are constantly arranging themselves as they respond to sea waving and ocean continuous motion [1]. In controlled, laboratory conditions, there are several mechanisms that influence tracers' assembly into patterns. Pattern formation has been attributed to particle properties, as wetting [2-5], surface filling fraction [3,6], or inertial effects [7]. But tracers can also be steered by underlying flows [8-11]. Even in controlled settings as standing Faraday waves, several mechanisms lead to fluid motion decoupled from the waves' periodicity $[12,13]$, where tracers' motion becomes difficult to predict. An example is the simple case of propagating waves generated by an oscillating plunger, which has shown a surprising flow reversal when crosswise waves form $[14,15]$. In particular, walls play a relevant role, because dynamic wetting between liquid and moving walls determines flow boundary conditions [16-19] and strongly influences dissipation [20-22].

Published by the American Physical Society under the terms of the Creative Commons Attribution 4.0 International license. Further distribution of this work must maintain attribution to the author(s) and the published article's title, journal citation, and DOI.
In this Letter, we identify a new mechanism leading to streaming at the interface of standing Faraday waves: a space-time-dependent shear produced at the oscillatory liquid-wall contact line. The steadiness and localization of the induced streaming drives surface tracers to a periodic array of vortices and then, as the Faraday-wave amplitude increases, to sharp rotating rings and to a spiking pattern, which we name the hedgehoglike pattern, all documented and explained here for the first time.

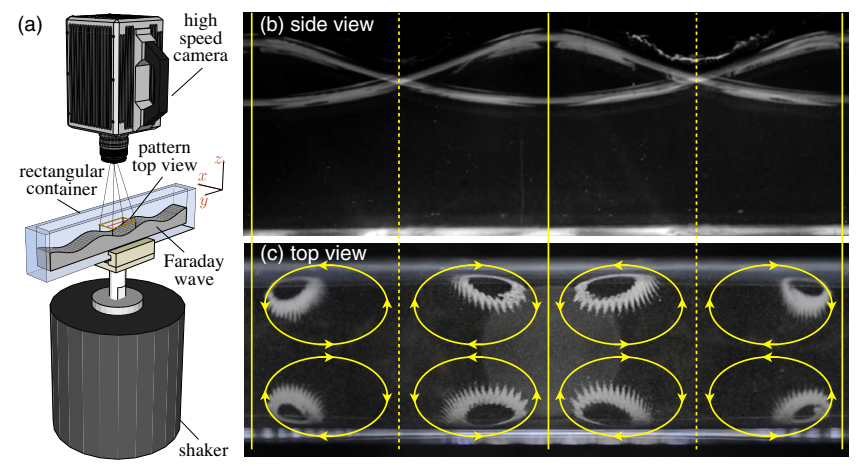

FIG. 1. Experimental setup and hedgehoglike pattern. (a) An electromechanical shaker vibrates vertically the acrylic container with a water-surfactant liquid mixture and a small amount of silver-coated hollow glass microspheres. Side (b) and top (c) views of the patterns observed on a single Faraday wavelength. Solid (dashed) lines indicate antinodes (nodes). (c) Arrows depict streaming flows. 
This Letter is organized as follows: We start by presenting the Faraday-wave setup and then the tracers' self-organized patterns. After, we focus on measurements of the streaming flow, which we hypothesize is produced by a shear gradient at contact lines. We replicate this flow with a simplified setup that engenders only shearing of the liquid at the solid wall. We finish by presenting a $2 \mathrm{D}$ compressible advection model that considers the minimal ingredients in the Faraday experiment, which reproduces the observed patterns.

Faraday-wave experiment.-The setup used to produce Faraday waves is a rectangular acrylic container with interior dimensions of $26.5 \times 280 \times 55 \mathrm{~mm}^{3}$ attached to an electromechanical shaker allowing vertical vibrations [Fig. 1(a)]. The container is filled up to $30 \mathrm{~mm}$ deep with an aqueous solution of $218 \mathrm{~g}$ of distilled water and $2 \mathrm{ml}$ of Kodak Photo-Flo, used as wetting agent [23], and sprinkled homogeneously with $0.1 \mathrm{mg}$ of $10 \mu \mathrm{m}$-diameter silvercoated hollow glass microspheres, whose mass density is $\rho=1.4 \mathrm{~g} \mathrm{~cm}^{-3}$ (Dantec Dynamics, S-HGS). The container vibrates sinusoidally $z(t)=a \sin \left(\omega_{d} t\right)$, at driving amplitude $a$ and fixed frequency $f_{d}=\omega_{d} / 2 \pi=8.30 \mathrm{~Hz}$. This driving induces supercritical (nonhysteretic) Faraday waves at $f=f_{d} / 2=4.15 \mathrm{~Hz}$, above a normalized critical acceleration amplitude $\Gamma_{c}=a_{c} \omega_{d}^{2} / g=0.144 \pm 0.001(g$ is the gravitational acceleration). Under these experimental conditions, the observed stationary Faraday wave forms a $(3,0)$ mode, i.e., three longwise wavelengths with no crosswise component.

To characterize the flow, we measured simultaneously the tracer positions and the wave amplitude. To track the tracers, we set a camera above the container and a focused razing horizontal light from the side. The recording frequency is 100 frames per second with a $1920 \times 1080$ pix $^{2}$ resolution. The local height of Faraday waves is simultaneously measured from a side view using a tilted mirror [Fig. 1(b)].

Flow structure and tracers' self-organization.-As soon as Faraday waves emerge, tracers at the interface begin to circulate inside cells limited in the $x$ direction by the container central plane and the walls and in the $y$ direction by node and antinode planes, as indicated in Fig. 1(c). The circulation is steady, and its origin will be discussed later. Cells adjacent to antinodes or center lines shelter identical but counterrotating patterns, as shown in Fig. 1(c). Tracers near walls always go from node to antinode [24]. This circulation-cell structure is compatible with previous bulk streaming flow observations [18].

Tracers display four organization regimes depending on the Faraday-wave amplitude $A$, as shown in Fig. 2(a) and Ref. [24]: (i) For $A \leq 1.5 \mathrm{~mm}$, the rotating tracers spread homogeneously over each cell, forming a periodic array of vortices, (ii) above $A \approx 1.5 \mathrm{~mm}$, tracers shrink into rotating rings, due to the flow three-dimensional nature, which induces an apparent compressibility at the interface, (iii) for $A>2.8 \mathrm{~mm}$, the rotating ring sharpens and exhibits spikes in a shape that is reminiscent of a hedgehog. The spikes'
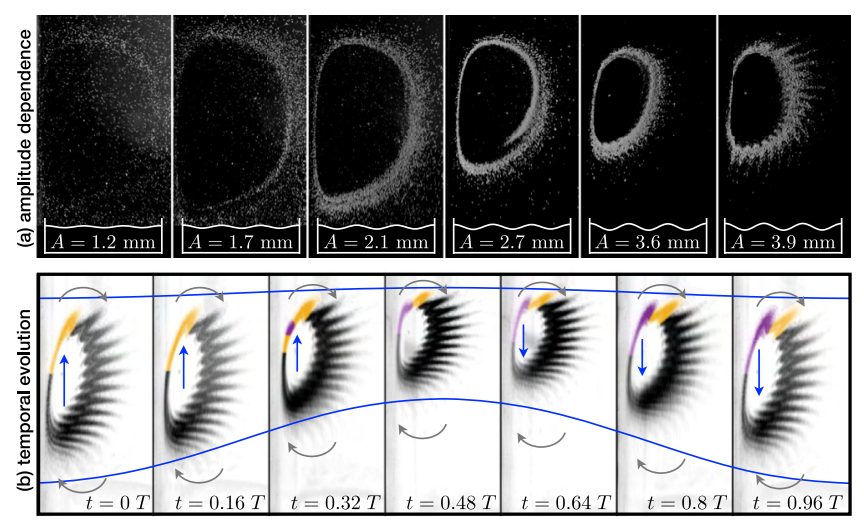

FIG. 2. Elementary hedgehoglike pattern. (a) Pattern at a given phase for various wave amplitudes $A$. (b) Breathing motion during a single oscillation (inverse gray scale); the formation of a new spike is highlighted (purple).

length increases with $A$. (iv) Finally, for $A>4.3 \mathrm{~mm}$, spikes become very long and oscillate in a timescale much larger than the Faraday waves', and each cell interacts with its neighbors (see videos in Supplemental Material [24]).

In hedgehoglike patterns, one new spike emerges during each oscillation period. The spike formation can be observed along a representative cycle shown in Fig. 2(b). In one hand, the pattern compresses and stretches periodically slaved to free-surface oscillations. This induces a back-and-forth breathing motion during the oscillation period, as schematized with vertical arrows in Fig. 2(b). On the other hand, the steady streaming slowly rotates the pattern, as shown with the curved arrows in the same figure. Spikes appear during surface compression: Tracers near the wall are traveling faster than their counterparts near the antinode. Therefore, fluid parcels fold, giving birth to a spike.

To evaluate the role of the particle nature of tracers in pattern formation, we ran the experiment using soluble dyes (ink), and yet patterns remained. Moreover, increasing the tracer concentration by a factor of 5 keeps the observed patterns unaltered. These observations demonstrate that patterns are generated by the flow itself, and tracer interaction or buoyancy are not relevant, contrary to what was observed in larger-particle experiments [2,3].

Steady streaming.-In our experiments, we observe a steady circulation, as schematized in Fig. 1(c). Because of their small size, tracers follow fluid parcels. To characterize their displacement, we use image cross-correlation. However, due to high wave amplitudes, large in-plane deformation limits global measurements, specifically as the interface stretches due to the longitudinal wave. Therefore, we focused on eight small sections of the ring, shown in Fig. 3(a). We track the longitudinal in-plane displacement due to the Faraday wave as in a Lagrangian frame of reference. The time-dependent velocity of this frame of reference is obtained by linear interpolation along $y$ between the velocities of the two extremities of the ring. 

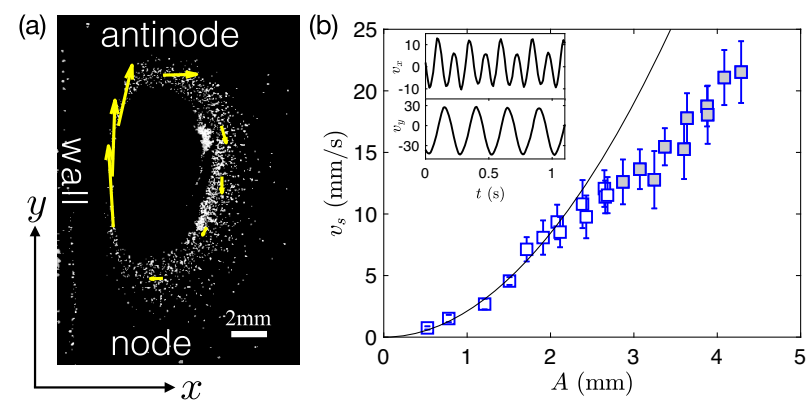

FIG. 3. (a) Tracer velocities around a unit-cell rotating ring, for $A=2.1 \mathrm{~mm}$. Streaming velocities, shown as arrows, are measured at eight different locations around the ring, being much larger close to the wall. (b) Streaming speed averaged over the three near-wall points as a function of wave amplitude. Solid points correspond to measurements with well-developed spikes along the ring. The solid line is a quadratic fit for $A<2.4 \mathrm{~mm}$. Inset: Examples of instantaneous velocity components at a given point around the ring.

Then we compute image cross-correlation between small windows that follow this frame of reference. The total velocity is deduced from the prescribed Lagrangian displacement plus the one measured from image crosscorrelation. Figure 3(a) shows an instantaneous picture, and the inset in Fig. 3(b) shows the time evolution of the $x$ and- $y$-velocity components of a single point near the wall.

Temporal evolution in $v_{y}$ shows subharmonic oscillations, whereas $v_{x}$ exhibits a harmonic mode. While the Faraday wave is the dominant motion along $y$, the $x$ direction is more sensitive to meniscus waves. Both velocity components have a well-defined nonzero time average, corresponding to the steady streaming. To evaluate this magnitude as a function of $A$, we define $v_{s}$ as the average speed between the three points near the wall. The main panel in Fig. 3(b) shows that $v_{s}$ increases first quadratically and then nearly linearly with $A$.

The final and most remarkable feature of the streaming flow is its heterogeneity along the ring. Indeed, Fig. 3(a) shows that the velocity is around 6 times larger near the walls than close to the container's center line. Therefore, near the wall, the liquid experiences a large acceleration linked to a strong shear gradient. For standing waves, due to the dynamical variation of the surface elevation between nodes and antinodes, a space-time-dependent shear gradient is ubiquitous whenever walls are present. Are circulating streaming flows a generic feature when a space-time variable shear gradient is exerted on a liquid-solid-air contact line? We answer this by isolating the oscillatory shear gradient.

Rotating disk experiment.-A circular disk (radius $R=40 \mathrm{~mm}$ ) rotates periodically between angles $\theta$ and $-\theta$, driven by a stepper motor [see Fig. 4(a)]. The disk is partially immersed in water up to its center, so that any point oscillates with an amplitude $r \theta$, with $r$ the distance from the center and with a maximum arc amplitude $A=R \theta$. We limited $A$ to $8 \mathrm{~mm}$ in order to avoid sloshing
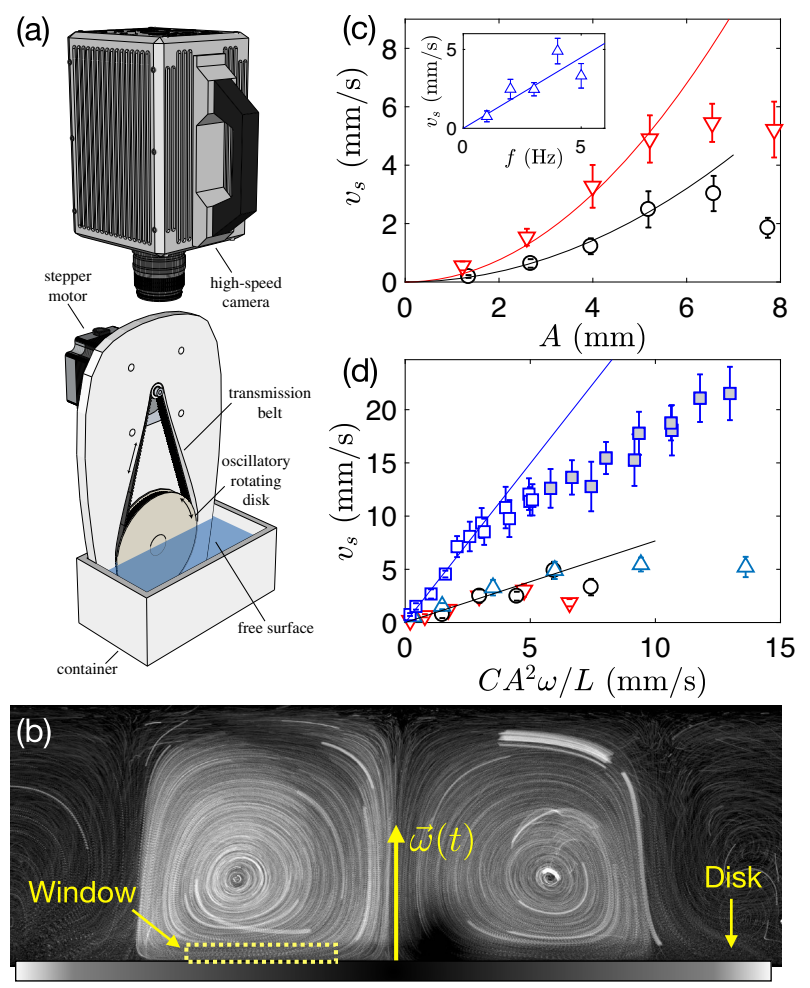

FIG. 4. (a) Schematic illustration of the rotating disk setup. (b) Top view of the observed streaming pattern, $A=R \theta=$ $2.67 \mathrm{~mm}$ and $f=2 \mathrm{~Hz}$. The dashed-line rectangle shows the PTV window. (c) Streaming speed as a function of disk oscillation amplitude $A$, for $f=2 \mathrm{~Hz}$ (Down pointing triangle) and $f=4 \mathrm{~Hz}$ (Circle). Inset: $v_{s}$ linear frequency dependence for fixed $A$. (d) $v_{s}$ as a function of $C \omega A^{2} / L$, with $L=R$ and $C=$ 0.35 for the disk and $L=\lambda$ and $C=2.5$ for Faraday data. The disk data collapse on a single curve, saturating at higher values. For both experiments, an $A^{2}$ scaling is observed for the loweramplitude regime, shown as straight lines.

motion. Oscillation frequency $f$ is set independently. The disk is made of acrylic, having similar wetting properties as the Faraday-wave experiment. The disk oscillatory motion, therefore, simulates the spatiotemporally varying triple-line position distinctive of Faraday waves.

Figure 4(b) presents a digitally overexposed image, revealing flow streamlines. The result is striking, as it shows steady vortices reminiscent of those observed in the Faraday experiment for low amplitudes. In order to further characterize this steady streaming, we measured the spatial average streaming speed $v_{s}$ in a window close to the disk [also shown in Fig. 4(b)]. Here, because motion is much slower than in the Faraday experiment, we are able to perform simple particle tracking velocimetry (PTV). The streaming speed is then computed as a spatiotemporal average from individual tracer measurements. We performed measurements for fixed $f$ increasing $A$ and also for fixed $A$ increasing $f$. We observe a linear increase of $v_{s}$ with $f$ and a quadratic dependence on $A$, with a saturation at the highest values [Fig. 4(c)]. When plotting $v_{s}$ as a 
function of $A^{2} \omega / R$, all disk measurements collapse on a single curve [see Fig. 4(d)]. The consistency of this scaling law in both experiments at low amplitudes allows us to conclude that the circulation comes from the verticalvelocity shear gradient at the walls, similar to streaming laws in Refs. [18,25] but specifically applied to the contact line. Indeed, the data are consistent with $v_{s} \propto\left\langle w_{\sim} \partial_{y} w_{\sim}\right\rangle /(2 \pi f)$, where the vertical velocity component of the contact line at the wall is $w_{\sim}=$ $2 \pi f A \sin (k y) \cos (2 \pi f t)$ for Faraday waves and $w_{\sim}=$ $2 \pi f A y \cos (2 \pi f t) / R$ for the disk. Here, the brackets \langle\rangle stand for spatiotemporal average in each case. The constant $C$ used in Fig. 4(d) is the result of the numerical factors for each case, considering also the spatial averaging. The law $v_{s} \propto\left\langle w_{\sim} \partial_{y} w_{\sim}\right\rangle /(2 \pi f)$ also gives an explanation to the generation of eddies on the crosswise waves in Refs. [14,15], which share the common feature of sheared oscillating contact lines, despite the different nature of the generated waves (gravity vs capillary). It also consistently predicts that nodes (antinodes) generate inward (outward) jet streams in such a configuration.

Simple 2D compressible advection model.-The experiment with the oscillatory rotating disk successfully isolates the gradient of shear exerted on the contact line as it oscillates on the wall. It clearly shows that the primary origin of the circulation of the observed patterns in Faraday waves is not the presence of the wave itself but the shear gradient along the contact line. The question that naturally arises is whether it is possible to go further with a model that is able to predict not only a rotating velocity field but also complex hedgehoglike structures.

We have concluded that there are three fundamental features required for hedgehoglike-structure formation, which can be put into a phenomenological 2D compressible advection model. These are

(i) A steady circulation.-To build a velocity field that has the features observed in Faraday cells, we consider a modification of the classic flow of a 2D rectangular cavity driven by a moving wall. Instead of imposing a uniform velocity at one of the boundaries of the cavity, we imposed a nonuniform velocity, $\propto-\sin (2 k y)$ in the lower boundary at $x=0$, to mimic the sheared contact line. Free-slip boundary conditions are applied on the other boundaries, as they correspond to symmetry planes of Faraday-wave cells. The streamlines due to the presence of this flow can be calculated analytically [24] and are shown in Fig. 5(a).

(ii) A periodic stretching.-Figure 2(b) shows that structures undergo periodic stretching in time. To account for this, we added an oscillatory field given by $v(x, y, t)=-\epsilon \sin k y \cos \omega t$, where $\epsilon$ is the amplitude of the deformation due to the main motion of the stationary Faraday wave as shown in Fig. 5(b) for a particular phase of the oscillatory motion.

(iii) A steady converging field.-This flow accumulates the particles into a ring [see Fig. 5(c)]. To account for this,

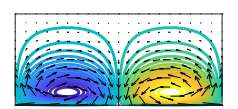

(a) circulation

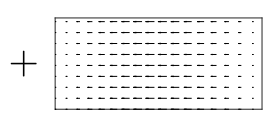

(b) stretching (c) converging
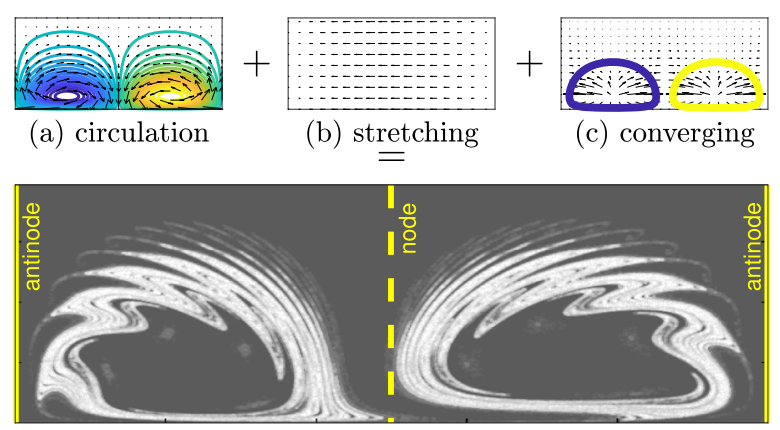

(d) hedgehoglike structure

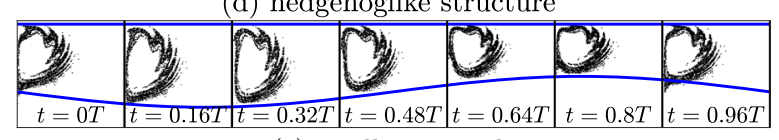

(e) oscillation cycle

FIG. 5. (a) Steady circulation, (b) periodic stretching, and (c) a steady converging field featured by a ring are the basic ingredients to generate hedgehoglike structures. The superposition of the velocity fields allows agglomeration of particles (d) and similar dynamics, including rotation, breathing, and formation of spikes in every cycle (e) as those observed on Faraday waves.

we took the rotating velocity field and manipulated it to generate a secondary flow that converges particles into one of its streamlines [24].

The two last ingredients allow tracers' clustering through the apparent compressibility of the free-surface flow developed by three-dimensional effects. These are very weak in the disk experiment, which explains the absence of sharp rings and spikes.

We ran simple numerical simulations in which an initially random distribution of particles is advected by the superposition of these three velocity fields. As shown in Supplemental Material [24], particles agglomerate into structures that are remarkably similar to those observed on Faraday waves [Fig. 5(d)]. The model captures successfully very specific and complex features of the motion of the structures: the breathing dynamics [Fig. 5(e)], the mechanism for generation of spikes in each period, and a gradual decrease of the length of the spikes as they approach the wall.

In conclusion, we have found experimentally that the flow at the interface of Faraday waves undergoes a transition from a periodic array of vortices with homogeneous distribution of tracers to rotating rings and then to spiking, hedgehoglike patterns as the wave amplitude increases. The observed spikes are created at each period due to the strong shear gradient at the wall and the fast deceleration of the circulating ring, away from the walls. We demonstrated that the main source of the streaming flow is the spatiotemporal-dependent shear on the contact line at the wall boundary, induced by the Faraday wave itself. The streaming flow is fairly generic, as a similar circulation flow is observed in the oscillating rotating disk experiment. Both experiments follow the scaling $v_{s} \propto$ $\left\langle w_{\sim} \partial_{y} w_{\sim}\right\rangle /(2 \pi f)$ for the average streaming speed near 
the wall, where $w_{\sim}$ is the contact-line vertical velocity component on the wall. The proposed scaling law provides also an explanation to the eddies and jet streams documented in Refs. [14,15], setting the basis for the understanding of flow reversal on propagating waves generated by oscillating plungers. Finally, we propose a simplified model that considers the minimal ingredients to obtain hedgehoglike patterns, namely, the stationary circulation, the stretching component due to the oscillation, and a steady converging field. The model imitates the patterns observed experimentally in many regards. A quantitative model encompassing the three-dimensional flow remains to be studied to explain the transitions. As a perspective, the flow will benefit from being studied under the framework of Lagrangian coherent structures [1,26], which should provide more information about the patterns' dynamic features. This could also help us to understand how the stirring process, conducted by a simple periodic flow with a nonlinearly generated stationary component, can lead to such tracers' complex patterns with still a high degree of spatiotemporal organization.

H. A. acknowledges the support of Fondecyt postdoctoral program, Grant No. 3160341. L. G., P. G., and N. M. acknowledge support of Agencia Nacional de Investigación y Desarrollo (ANID) through the Fondecyt Grants No. 11170700, No. 11191106, and No. 1180636, respectively. We also acknowledge an anonymous referee for suggesting the name for the spiking pattern.

*hector.alarcon@uoh.cl

pablo.gutierrez@uoh.cl

*leonardo.gordillo@usach.cl

[1] G. Haller, Lagrangian coherent structures, Annu. Rev. Fluid Mech. 47, 137 (2015).

[2] G. Falkovich, A. Weinberg, P. Denissenko, and S. Lukaschuk, Surface tension: Floater clustering in a standing wave, Nature (London) 435, 1045 (2005).

[3] C. Sanl1, D. Lohse, and D. van der Meer, From antinode clusters to node clusters: The concentration-dependent transition of floaters on a standing Faraday wave, Phys. Rev. E 89, 053011 (2014).

[4] M.-J. Dalbe, D. Cosic, M. Berhanu, and A. Kudrolli, Aggregation of frictional particles due to capillary attraction, Phys. Rev. E 83, 051403 (2011).

[5] D. Vella, Floating versus sinking, Annu. Rev. Fluid Mech. 47, 115 (2015).

[6] M. Berhanu and A. Kudrolli, Heterogeneous Structure of Granular Aggregates with Capillary Interactions, Phys. Rev. Lett. 105, 098002 (2010).

[7] F. Santamaria, G. Boffetta, M. Martins Afonso, A. Mazzino, M. Onorato, and D. Pugliese, Stokes drift for inertial particles transported by water waves, Europhys. Lett. 102, 14003 (2013).

[8] G. Boffetta, J. Davoudi, B. Eckhardt, and J. Schumacher, Lagrangian Tracers on a Surface Flow: The Role of Time Correlations, Phys. Rev. Lett. 93, 134501 (2004).
[9] J. R. Cressman, J. Davoudi, W. I. Goldburg, and J. Schumacher, Eulerian and Lagrangian studies in surface flow turbulence, New J. Phys. 6, 53 (2004).

[10] P. Gutiérrez and S. Aumaitre, Clustering of floaters on the free surface of a turbulent flow: an experimental study, Eur. J. Mech. B 60, 24 (2016).

[11] S. Lovecchio, C. Marchioli, and A. Soldati, Time persistence of floating-particle clusters in free-surface turbulence, Phys. Rev. E 88, 033003 (2013).

[12] N. Francois, H. Xia, H. Punzmann, S. Ramsden, and M. Shats, Three-Dimensional Fluid Motion in Faraday Waves: Creation of Vorticity and Generation of Two-Dimensional Turbulence, Phys. Rev. X 4, 021021 (2014).

[13] S. V. Filatov, V. M. Parfenyev, S. S. Vergeles, M. Y. Brazhnikov, A. A. Levchenko, and V. V. Lebedev, Nonlinear Generation of Vorticity by Surface Waves, Phys. Rev. Lett. 116, 054501 (2016).

[14] H. Punzmann, N. Francois, H. Xia, G. Falkovich, and M. Shats, Generation and reversal of surface flows by propagating waves, Nat. Phys. 10, 658 (2014).

[15] V. Aleksandrov, S. Kopysov, and L. Tonkov, Excitation of vortex flows on the free surface of a liquid by a vibrating plate, Tech. Phys. 64, 939 (2019).

[16] C.-L. Ting and M. Perlin, Boundary conditions in the vicinity of the contact line at a vertically oscillating upright plate: an experimental investigation, J. Fluid Mech. 295, 263 (1995).

[17] L. Gordillo and N. Mujica, Measurement of the velocity field in parametrically excited solitary waves, J. Fluid Mech. 754, 590 (2014).

[18] N. Périnet, P. Gutiérrez, H. Urra, N. Mujica, and L. Gordillo, Streaming patterns in Faraday waves, J. Fluid Mech. 819, 285 (2017).

[19] Y. Huang, C. L. P. Wolfe, J. Zhang, and J. Q. Zhong, Streaming controlled by meniscus shape, J. Fluid Mech. 895, A1 (2020).

[20] L. Jiang, M. Perlin, and W. W. Schultz, Contact-line dynamics and damping for oscillating free surface flows, Phys. Fluids 16, 748 (2004).

[21] L. Gordillo and M. A. Garcia-Ñustes, Dissipation-Driven Behavior of Nonpropagating Hydrodynamic Solitons Under Confinement, Phys. Rev. Lett. 112, 164101 (2014).

[22] B. Dollet, É. Lorenceau, and F. Gallaire, Transition from Exponentially Damped to Finite-Time Arrest Liquid Oscillations Induced by Contact Line Hysteresis, Phys. Rev. Lett. 124, 104502 (2020).

[23] J. Wu, R. Keolian, and I. Rudnick, Observation of a Nonpropagating Hydrodynamic Soliton, Phys. Rev. Lett. 52, 1421 (1984).

[24] See Supplemental Material at http://link.aps.org/ supplemental/10.1103/PhysRevLett.125.254505 for experimental videos, technical details, and numerical simulations of the 2D compressible advection model.

[25] J. A. Nicolás and J. M. Vega, Three-dimensional streaming flows driven by oscillatory boundary layers, Fluid Dyn. Res. 32, 119 (2003).

[26] M. Farazmand and G. Haller, Computing Lagrangian coherent structures from their variational theory, Chaos 22, 013128 (2012). 